\title{
MEASURED PROPERTIES OF THE DUVFEL HIGH BRIGHTNESS, ULTRASHORT ELECTRON BEAM
}

\author{
W.S. Graves, G.L. Carr, L.F. DiMauro, A. Doyuran, R. Heese, E.D. Johnson, S. Krinsky, C. \\ Neuman, G. Rakowsky, J. Rose, J. Rothman, J. Rudati, T. Shaftan, B. Sheehy, J. Skaritka, L.-H. Yu \\ NSLS, Brookhaven National Laboratory, Upton, NY 11973, USA \\ D. H. Dowell, Boeing Physical Sciences Research Center, Seattle, WA 98124 USA \\ P. Emma, SLAC, Stanford, CA 94025, USA
}

\begin{abstract}
The DUVFEL electron linac is designed to produce sub-picosecond, high brighness electron bunches to drive an ultraviolet FEL. The accelerator consists of a 1.6 cell S-band photoinjector, variable pulse length Ti:Sapp laser, 4 SLAC-type S-band accelerating sections, and 4-dipole chicane bunch compressor. In preparation for FEL operation, the compressed electron beam has been fully characterized. Measurement of the beam parameters and simulation of the beam are presented.
\end{abstract}

\section{PHOTOINJECTOR}

Table 1: DUVFEL photoinjector properties

\begin{tabular}{|l|l|}
\hline \multicolumn{2}{|c|}{ Laser system } \\
\hline Type & Titanium:Sapphire \\
\hline IR laser energy & $30 \mathrm{~mJ}$ at $800 \mathrm{~nm}$ \\
\hline UV laser energy & $\begin{array}{l}300 \mathrm{uJ} \text { at } 266 \mathrm{~nm} \text { on } \\
\text { cathode }\end{array}$ \\
\hline Laser pulse length & Adjustable 1-5 ps FWHM \\
\hline & RF Cavity \\
\hline Type & $\begin{array}{l}\text { Modified 1.6 cell } \\
\text { BNL/SLAC/UCLA Gun IV }\end{array}$ \\
\hline Cathode material & Copper \\
\hline Maximum energy & $5.1 \mathrm{MeV}$ \\
\hline Loaded Q & 7100 \\
\hline Unloaded Q & 13700 \\
\hline RF pulse length & $3.5 \mathrm{us}$ \\
\hline Quantum efficiency & $\sim 1 . \mathrm{E}-5$ \\
\hline Vacuum & $\sim 8 . \mathrm{E}-9$ torr \\
\hline Timing jitter & $<1 \mathrm{ps}$ \\
\hline
\end{tabular}

The properties of the laser and photoinjector are summarized in Table 1. In typical running, $10 \mathrm{~mJ}$ of IR light is produced by the Spectraphyics Tsunami Ti:Sapphire oscillator and TSA50 amplifier, which is frequency tripled to produce $450 \mathrm{uJ}$ of UV light. After spatial filtering and aperturing of the gaussian mode to produce a nearly uniform laser spot, about $200-300 \mathrm{uJ}$ is delivered to the cathode. This produces $300 \mathrm{pC}$ of charge at the accelerating phase of 30 degrees. The RF cavity is a Gun IV [1] with copper cathode that has been modified for better performance [2]. In principle, the laser pulse length may be adjusted from 100 fs to 10 ps, however there are practical limitations on the range of adjustment due to dispersion characteristics and efficiency of the
BBO crystals. The thickness of the harmonic crystals is optimized for pulse lengths from 1-5 ps. Within this range of pulse lengths there is evidence [3] of variations in the time profile of the UV light that are sensitive to the phase-matching angle of the crystal.

\section{LINAC}

The linac consists of 4 SLAC S-band traveling wave sections with a maximum energy of $210 \mathrm{MeV}$. There is a four-magnet chicane [4] between linac tanks 2 and 3 to compress the electron beam to sub-ps bunch lengths. The photoinjector cavity and linac tanks 1 and 2 are powered by a single Thomson $45 \mathrm{MW}$ klystron. Each of these sections is independently phased with high power phase shifters. Tanks 3 and 4 are each powered by independent $45 \mathrm{MW}$ klystrons. A single klystron for the photoinjector and linac tanks before the chicane has the advantage of eliminating relative phase jitter and thus variations in bunch length exiting the chicane. The fast phase jitter of these sections relative to the laser arrival time is estimated to be approximately $0.5 \mathrm{ps}$.

Following the fourth linac section are a number of quadrupoles for matching into the NISUS undulator and two 70 degree dipoles that are used as an energy spectrometer and for bunch length measurement using the RF zero phasing method.
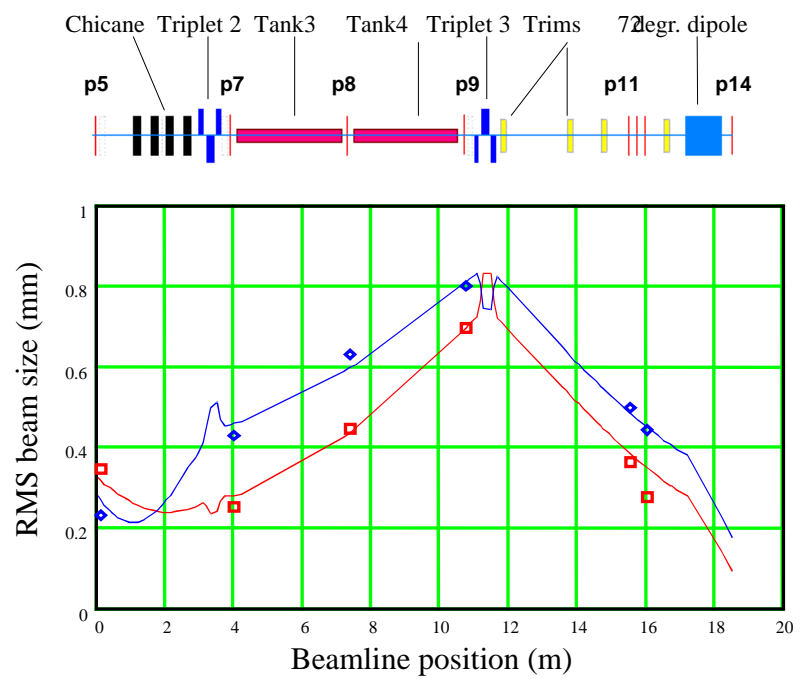

Figure 1: Comparison of simulated and measured beam sizes. 


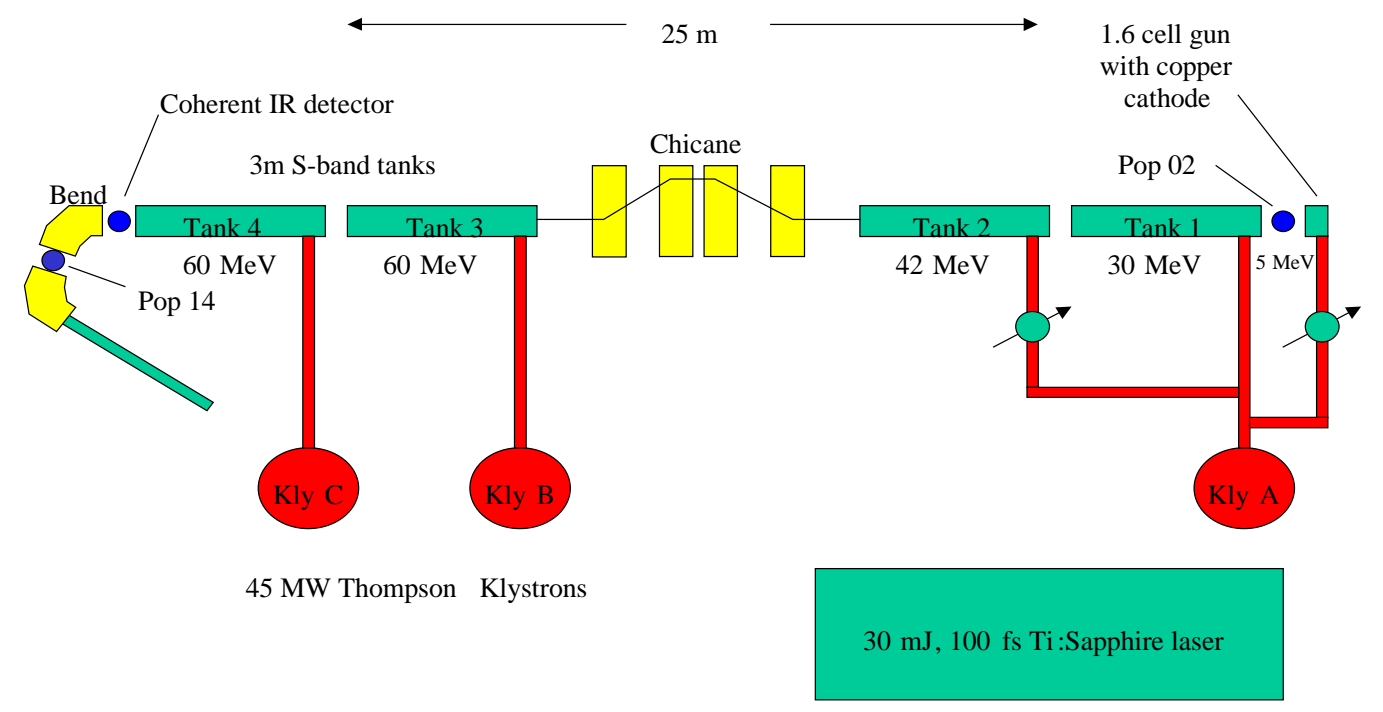

Figure 2: Layout of DUVFEL accelerator.

Figure 1 shows the evolution of beam size along the linac. The left edge of the figure is at the exit of tank 2, and the right edge is after the first 70 degree dipole. To perform this measurement, the beam size is measured at 6 points. An optimization using the MAD simulation code is then performed using the measured beam sizes as constraints, and varying the input Twiss parameters. The quadrupole strengths and linac settings are held fixed according to the measured values. The emittance calculated from this method generally agrees well with the emittance found from quadrupole scans performed at several locations. However, the optimization method appears more robust for accurate predictions of the lattice function beta and its derivative.

\section{COMPRESSED BEAM PARAMETERS}

The beam parameters vs bunch compression are shown in Fig 3. The parameters are plotted vs phase of tank 2, which creates the energy chirp for compression in the chicane. Zero phase corresponds to on-crest acceleration and no compression. Maximum compression occurs near 35 degrees off crest. At maximum compression the nominal beam parameters are peak current $=600 \mathrm{~A}$, RMS normalized emittances of $5.6 \mathrm{~mm}$-mrad horizontal and 5.0 mm-mrad vertical. The error bars on the current and bunch length measurements are the standard deviation of approximately 10 measurements performed in succession. The emittance error bars are derived from the variation in beam sizes during quadrupole scans. The emittance continues to increase as the bunch overcompresses and stretches. This is most likely due to generation of strong coherent synchrotron radiation in chicane magnets 2 and 3 where the bunchlength is minimum.

\section{SLICE EMITTANCE MEASUREMENT}

The RF-zero phasing bunch length measurement $[3,5]$ images the beam onto a scintillating screen in a dispersion region, and the horizontal extent of the beam is dominated by a correlated energy spread that produces the time profile. The slice emittance, and slice dynamics, of the beam can be measured by performing a quadrupole scan during the RF-zero phasing measurement that varies the vertical beam size about a waist. In this measurement,
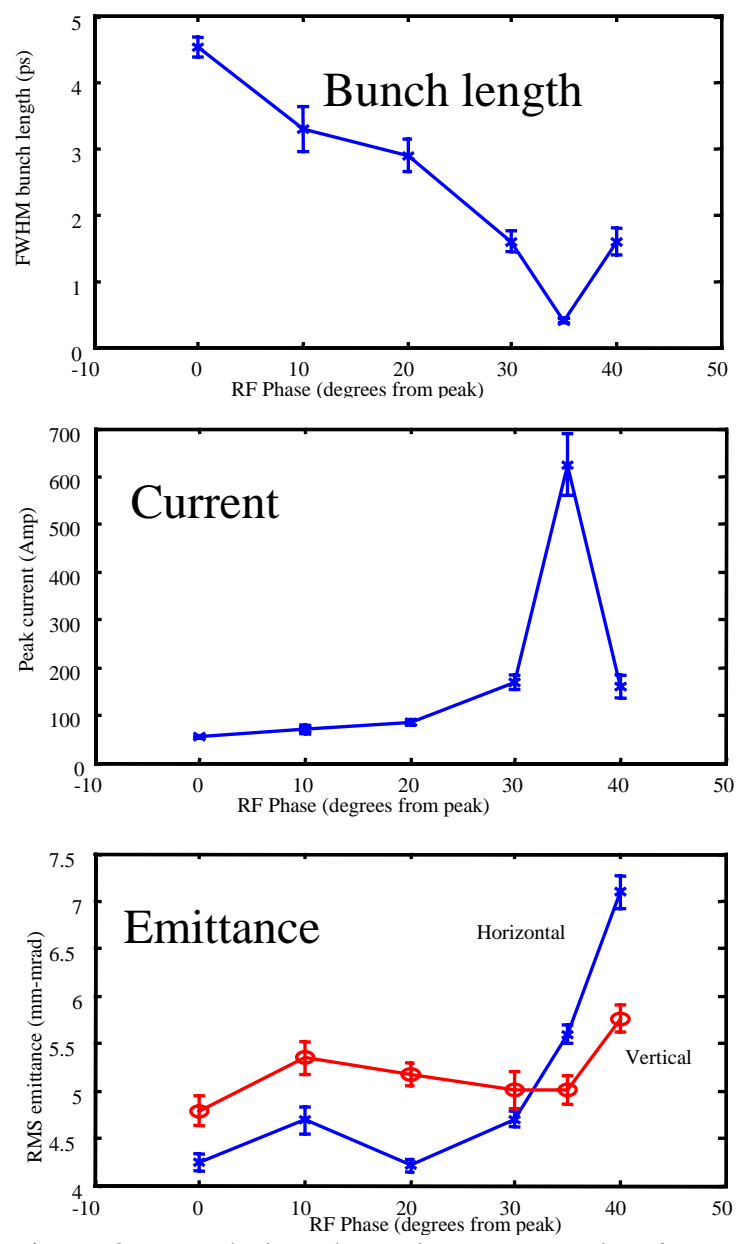

Figure 3: Bunch length, peak current, and emittance vs phase of tank 2. 

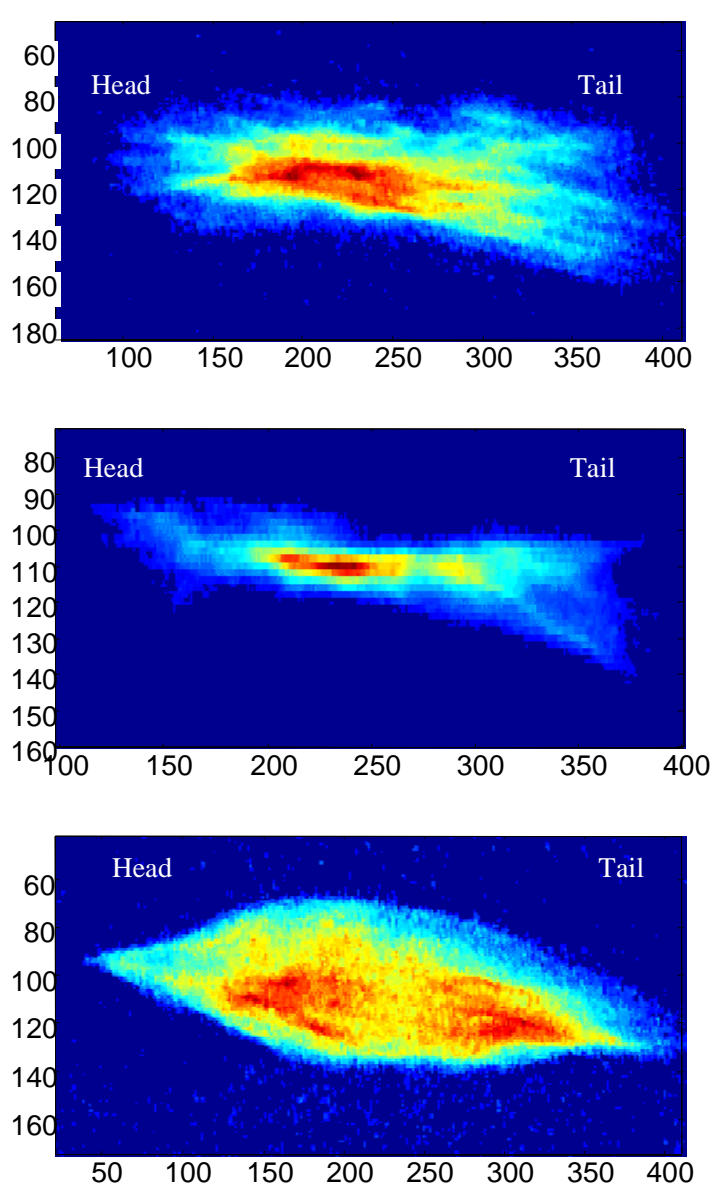

Figure 4: Quadrupole scan of electron beam during RFzero phasing measurement. Horizontal direction is time (FWHM is $\sim 4.5 \mathrm{ps}$ ). TOP: weak quad, beam converging vertically. MIDDLE: moderate quad strength, beam center at waist, ends still converging. BOTTOM: strong quad, beam center diverging, ends at waist.

the entire beam image is recorded in a single-shot, then software is used to slice the beam into thin time-slices. This is repeated for different quadrupole settings. The time resolution of the method is determined by the variation in horizontal betatron beam size during the quadrupole scan, and the need to fit the entire beam onto the scintillator (the energy spread must not be made too large).

Fig. 4 shows the beam image on the RF-zero phasing screen at 3 different quadrupole settings. It is clear that different time slices of the beam are at different betatron phase advances. This is due to varying space charge and $\mathrm{RF}$ forces at low energy (imperfect emittance compensation). For the images shown the time resolution is about $150 \mathrm{fs}$.

The images were cut into 10 equal width, 500 fs wide slices and each slice analysed. The resulting Twiss parameters of the slices are plotted in Fig. 5.
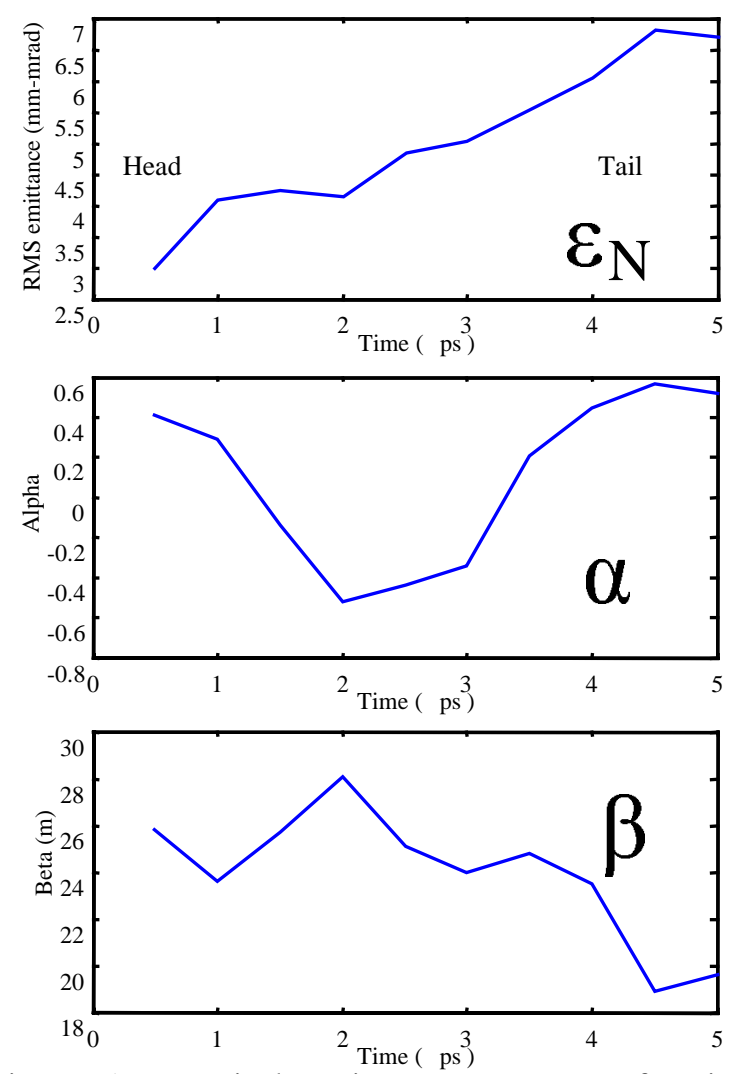

Figure 5: Vertical Twiss parameters as function of position in bunch. Slice width $=500$ fs. Note substantial variation in slice emittance from head to tail, and symmetry of alpha.

It is interesting to note that the emittance grows by a factor of two from the head to the tail of the bunch, that the beta function is almost constant, and that alpha has a large symmetric variation about the bunch center. For this measurement the charge was $200 \mathrm{pC}$, energy $=75$ $\mathrm{MeV}$.

\section{REFERENCES}

[1] D.T. Palmer et al, in Proceedings of the 1997 Particle Accelerator Conference (Vancouver, B.C., 1997), p. 2687

[2] J. Rose et al, in Proceedings of the 2001 Particle Accelerator Conference (Chicago, IL, 2001)

[3] W.S. Graves et al, "Ultrashort Electron Bunch Length Measurements at DUVFEL", in these proceedings (Chicago, IL, 2001)

[4] W.S. Graves et al, in Proceedings of the 1997 Particle Accelerator Conference (Vancouver, B.C., 1997), p. 1197

[5] D.X. Wang and G.A. Krafft, in Proceedings of the 1997 Particle Accelerator Conference (Vancouver, B.C., 1997), p. 2020. 